\title{
Klebsiella singaporensis
}

National Cancer Institute

\section{Source}

National Cancer Institute. Klebsiella singaporensis. NCI Thesaurus. Code C86459.

A species of facultatively anaerobic, Gram negative, straight rod shaped bacteria assigned to the phylum Proteobacteria. This species is nonmotile, oxidase negative, capsulated, non endosporeforming and produces isomaltulose from sucrose but does not produce indole. K. sing aporensis is found in soil, and its pathogenic potential is unknown. 